Journal of Computer Science 7 (7): 1119-1127, 2011

ISSN 1549-3636

(C) 2011 Science Publications

\title{
Fuzzy Logic Controller for Wireless Video Transmission
}

\author{
${ }^{1}$ K.P. Rajesh and ${ }^{2}$ K.S. Ramaswami \\ ${ }^{1}$ Department Master of Computer Applications, \\ Park College of Engineering and Technology, Coimbatore, India \\ ${ }^{2}$ Department Mathematics, \\ Coimbatore Institute of Technology, Coimbatore, India
}

\begin{abstract}
Problem statement: As the Variable Bit Rate (VBR) is high in MPEG, the video experienced long delay and unexpected data loss. In wireless channel, due to noise and interference of other signals transmission rate cannot be predicted. Approach: In this paper we proposed an intelligent fuzzy logic controller for transmission of Moving Picture Expert Group (MPEG-4) video signals over wireless channel. A Nero-Fuzzy (NF) controller was used to control the output rate of the buffer so that the signals were transmitted smoothly to wireless channel. Results: Simulation results showed that the use of intelligent fuzzy logic and nero-fuzzy controller improved the data transmission rate and decreased long delay when compared with other conventional methods. Conclusion: We proposed an intelligent fuzzy logic controller which adjusts the transmission rate dynamically so that the transmission loss and delay could be minimized. The traffic shaping buffer is used to prevent excess back-to-back transmission of video signals.
\end{abstract}

Key words: Moving Picture Expert Group (MPEG), Variable Bit Rate (VBR), Nero-Fuzzy (NF), Asynchronous Transfer Mode (ATM), Self-Organizing Fuzzy (SOF), Quality of Service (QOS), wireless video transmission, fuzzy logic, Constant Bit Rate (CBR)

\section{INTRODUCTION}

Video applications are the major sources of traffic in the present wireless network. Moving Picture Expert Group-4 (MPEG) Variable Bit Rate (VBR) is a preferred technique for wireless transmission as constant image quality is produced by it. MPEG-4 VBR video source have high bit rate and needs more bandwidth for transmission in wireless network (Chabaa and Zeroual, 2009). The segmentation technique is used in video, to enhance the transmission and user interaction, video sequence is decomposed in to different content-resolution levels (Sasilatha and Raja, 2009).

Transmitted video signals are affected due to the interference of other wireless signals and the data transfer rate is also affected due to retransmission. The use of intelligent fuzzy controller will adopt itself to the conditions and regulates the transmission speed, so that maximum data transfer rate is obtained.

Fuzzy logic is used to make decision to regulate the video signal transfer rate dynamically (Breven and ElTayeb, 2009). So for various researches have been carried out in the wireless video transmission. Using two intelligent leaky buckets to control the data transfer rate in an Asynchronous Transfer Mode (ATM), fuzzy controller had been implemented in the leaky bucket (Breven and El-Tayeb, 2009). For Connection Admission Control (CAD) in multimedia transmission over wireless network Hopfield neural network has been applied to get Quality Of Service (QOS). Simulation results shows that the algorithm can maximize resource utilization but resources where not shared effectively (Chabaa and Zeroual, 2009). The fuzzy adaptive transmission rate control for the frame transmission in wireless LANs focuses the received signal strength indicator, the frame error rate and the medium access control delay to formulate a correct decision (Kazemian, 2009). A fuzzy adaptive rate control prediction scheme was used to avoid congestion in high utilization, while maintaining a good quality of service in ATM (Chao et al., 2010).

Using a Self-Organizing Fuzzy (SOF) controller the transmission of real-time MPEG-4 VBR video sequences over an ATM network had been studied. The computer simulation results show that the use of SOF controller reduces excessive delay and data loss at the user-network interface as compared with a conventional method in ATM (Priakanth and Thangaraj, 2009).

Corresponding Author: K.P. Rajesh, Department Master of Computer Applications, Park College of Engineering and Technology, Coimbatore, India 


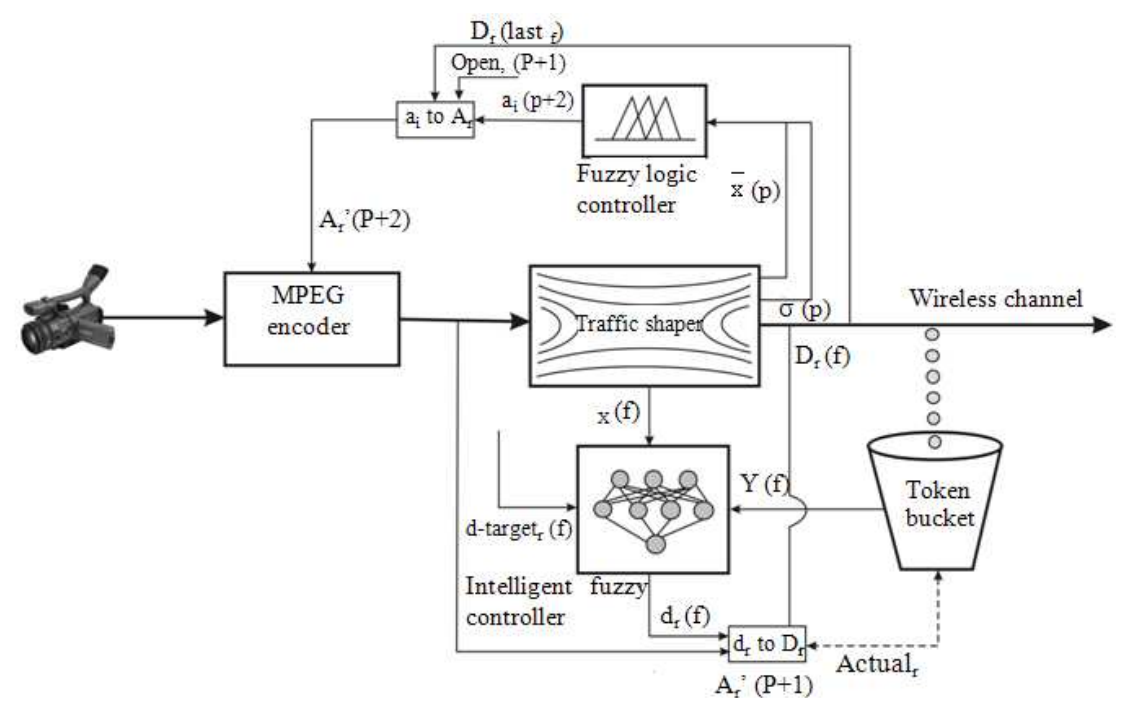

Fig. 1: Intelligent fuzzy logic controller for wireless video transmission

A fuzzy adaptive rate controller receives signal strength, the frame error rate and the medium access control delay to make a correct decision, simulation results demonstrates that the proposed scheme enhances the network throughput and the access delay (Elaal et al., 2010).

Wireless channel: The wireless channel has limited capacity and signal fades as it travels towards destination and the resource allocation in wireless channel is more complex than the wired domain. Heterogeneous media type with varying Quality Of Service (QOS) requires access to the wireless network. The media type categories are Real Time traffic (RT), such as voice and video are delay sensitive whereas Non Real-time Traffic (NRT) categories like text and still images are loss sensitive. In asynchronous transfer mode (ATM) the RT application is served by the class Constant Bit Rate (CBR) and Variable Bit Rate (VBR) and the NTR application is served by the class Available Bit Rate (ABR) (Samimi et al., 2009). A fuzzy resource controller can meet the objectives of simplifying resource allocation; the wireless channel utilization is improved by increasing the services to NRT sources.

Due to transmitter-receiver separation and the physical environment of the signal propagation path, the signal traveling between transmitter and receiver through a wireless medium undergo attenuation. The fading effect decreases the user's effective transmission rate, which results in increasing the need for retransmission by loss sensitive NRT traffic. When signals transmitted through wireless channel, the resulting fade type is determined by signals' and channel's characteristics. Signal parameters (bandwidth, signal interval) and channel parameters (multi-way time delay extension) determine that, different signal can experience different fading process (Mamdouhi et al., 2009). Traffic sources with lower effective rates may need to employ more robust channel coding, at the expense of their information transmission rates.

Development of fuzzy controller scheme for video transmission over wireless channel: The video transmission over wireless channel using RBF controller and NF controller is given in Fig. 1. As the diagram shows, in this paper a traffic-shaping buffer is introduced to manipulate and coordinate the VBR encoding video prior to entering the wireless channel.

The role of the shaper buffer is to smooth the video output traffic and to eliminate the burstiness of the video stream which enters the wireless network. The fuzzified mean value $\bar{\chi}(\mathrm{p})$ and the fuzzified standard deviation $\sigma(p)$ of the queue length from the trafficshaping buffer are given as input to the RBF controller. The output from the RBF controller is the fuzzified time-interval of arrival data ia $(p+2)$. The fuzzified queue length $\mathrm{X}(\mathrm{f})$ from the traffic-shaping buffer and the fuzzified available tokens from the generic cell rate algorithm commonly known as the leaky-bucket Y(f) are the inputs to the NF controller. The output from the $\mathrm{NF}$ controller is the fuzzified departure rate $\mathrm{d}_{\mathrm{r}}(\mathrm{f})$, where $\mathrm{f}$ denotes frame. The fuzzified values of $\bar{\chi}(\mathrm{p})$, $\sigma(p), i_{\alpha}(p+2), X(f), Y(f)$ and $d_{r}(f)$ are kept within the range of $(0 \ldots 1)$. 
In the computer simulation, based on the Phase Alternate Line (PAL), it is assumed that there are 24 frames per second and a GOP contains 12 frames, therefore there are 2 GOP's per second (Emmanuel et al., 2009). The $\mathrm{p}^{\text {th }}$ GOP is the picture group that has completely passed the network at any time instant. The $(p+1)^{\text {th }}$ GOP is the group which is in the process of passing through the model to reach the token-bucket and finally entering the wireless channel.

The MPEG encoder presently encodes the $(p+2)^{\text {th }}$ GOP. The token-bucket reserves the data only if the arrival rate is greater than the departure rate and the data is dropped if the memory space in the token-bucket is full. The departure rate's range $D_{r}(f)$ lies between the arrival rate $\mathrm{A}_{\mathrm{r}}(\mathrm{p}+1)$ and the actual token (transmission) rate actual ${ }_{r}$. On GOP by GOP basis the arrival rate $A_{r}$ $(p+1)$ is controlled whereas the departure rate $D_{r}(f)$ is controlled by frame by frame basis.

The block ' $d_{r}$ to $D_{r}$ ' calculates the departure rate $D_{r}$ (f) from the fuzzified departure rate $d_{r}$ (f) using the following equation:

$D_{r}(f)=d_{r}(f) \times\left(D_{r-m a x}-D_{r-m i n}\right)+D_{r-m i n}$

where, $d_{r}$ (f) is the fuzzified $D_{r}$ (f) and $D_{r-m i n}=$ $\min \left\{\mathrm{A}_{\mathrm{r}}(\mathrm{p}+1), \mathrm{a}_{\mathrm{rate}}\right\}$ and $\mathrm{D}_{\mathrm{r}-\max }=\max \left\{\mathrm{D}_{\mathrm{a}}(\mathrm{k}+1)\right.$, actual $\left.\mathrm{r}_{\mathrm{r}}\right\}$. The block ' $a_{i}$ to $A_{r}$ ' calculates the desired arrival rate $\hat{\mathrm{A}}_{\mathrm{r}}(\mathrm{p}+2)$ from the fuzzified time-interval of arrival data $\hat{a}_{i}(p+2)$ using the following proposio:

$$
\hat{\mathrm{A}}_{\mathrm{r}}(\mathrm{p}+2)=\frac{1}{\left[\hat{\mathrm{a}}_{\mathrm{i}}(\mathrm{p}+2) \times\left(\mathrm{A}_{\mathrm{i} \_ \text {max }}-\mathrm{A}_{\mathrm{i} \_ \text {min }}\right)+\mathrm{A}_{\mathrm{i} \_ \text {min }}\right]}
$$

where, $\mathrm{A}_{\mathrm{i}_{\_} \min }$ and $\mathrm{A}_{\mathrm{i}_{\_} \max }$ are defined as if $\operatorname{Open}_{\mathrm{r}}(\mathrm{p}+1)<$ $\mathrm{D}_{\mathrm{r}}\left(\right.$ Last $\left._{\mathrm{f}}\right)$ :

$$
\begin{aligned}
& \mathrm{A}_{\mathrm{i}_{-} \min }=\frac{1}{1.2 \text { actual }_{\mathrm{r}}} \\
& \mathrm{A}_{\mathrm{i}_{-} \text {max }}=\frac{1.2}{\text { actual }_{\mathrm{r}}} \\
& \text { if }\left(\frac{1}{\mathrm{~A}_{\mathrm{i}_{-} \max }}<\operatorname{Open}_{\mathrm{r}}(\mathrm{p}+1)<\frac{1}{\mathrm{~A}_{\mathrm{i}_{-} \min }}\right) \\
& \mathrm{A}_{\mathrm{i} \_ \text {max }}=\frac{1}{\operatorname{Open}_{\mathrm{r}}(\mathrm{p}+1)}
\end{aligned}
$$

Else:

$$
\begin{aligned}
& \mathrm{A}_{\mathrm{i} \_ \text {min }}=\frac{1}{\operatorname{Open}_{\mathrm{r}}(\mathrm{p}+1)} \\
& \mathrm{A}_{\mathrm{i} \_\max }=\frac{1}{\text { actual }_{\mathrm{r}}}
\end{aligned}
$$

The input to block ' $a_{i}$ to $A_{r}$ ' are the departure rate of the last frame in the $p^{\text {th }}$ GOP $D_{r}\left(\right.$ last $\left._{f}\right)$ and the estimated data rate of the $(p+1)^{\text {th }}$ GOP Open $n_{r}(p+1)$.

The traffic-shaper releases two sources of data. The MPEG encoder compressed video sequence and the data kept in the traffic-shaper. Suppose the data currently kept in the traffic-shaper is released within one GOP period and deliver video sequence without delay. Then the data send rate is:

To-send $_{r}(f)=A_{r}(p+1)+\frac{x(f)}{T_{G O P}}$

Where:

$\mathrm{x}(\mathrm{f})=$ The queue length in the traffic-shaper

$\mathrm{T}_{\mathrm{GOP}}=$ The time period of a GOP

Some of the data will be kept in the token bucket, if To-send $\mathrm{r}_{\mathrm{r}}$ is larger than $\mathrm{a}_{\text {rate }}$. So that the maximum value that $D_{r}$ could be without causing data loss is:

Available $_{r}(f)=\operatorname{actual}_{r}(f)+\frac{y(f)}{T_{\text {frame }}}$

Where:

$\mathrm{y}(\mathrm{f})=$ The amount of remaining memory space in the token bucket

$\mathrm{T}_{\text {frame }}=$ The time period of a frame:

D_target $_{r}(f)=\min \left(\right.$ To-send ${ }_{r}$, Available $\left._{r}\right)$

Given the reference data value D_target $(\mathrm{f})$ or the fuzzified targeted value of d_target $\mathrm{r}_{\mathrm{r}}$ (f) from Fig. 1 is calculated by rearranging (1):

$d_{-} \operatorname{target}(f)=\frac{D_{-} \max _{r}-D_{-} \min _{r}}{D_{-} \operatorname{target}(f)-D_{-} \min _{r}}$

If the range of $d_{-}$target $t_{r}$ is outside the range of (0..1), the boundary value is used.

NF Adaptive scheme: The NF controller is incorporated with the adaptive control scheme. A neural network represents an RBF1 controller, it has a three- 
layered architecture and uses fuzzy sets as its weight at the input and output layers. Fuzzy IF-THEN rules are present at the hidden layer. Multilayer neural networks back-propagation procedure is used to train the fuzzy membership functions. Through the learning process the parameter associated with the membership function changes, so that the network interprets the desired input-output map of the controller. The parameters got from the training procedure are inputted to the fuzzy system to make best control performance. The NF controller uses the Sugeno or Takagi-Sugeno-Kang method, introduced in 1985 (Lokman et al., 2010). The proposed Sugeno RBF1 controller outputs first-order membership function.

If $\mathrm{x}$ is $\mathrm{A}$ and $\mathrm{y}$ is $\mathrm{B}$ then:

$$
\mathrm{z}(\mathrm{x}, \mathrm{y} ; \mathrm{m}, \mathrm{n}, \mathrm{o})=\mathrm{m} * \mathrm{x}+\mathrm{n} * \mathrm{y}+\mathrm{o}
$$

where, $\mathrm{A}$ and $\mathrm{B}$ are the past history, while $\mathrm{m}, \mathrm{n}$ and $\mathrm{o}$ are all constants.

Because of the linear characteristic of each rule on the system's input variables, the Sugeno method is ideal for acting as an interpolating supervisor of multiple linear controllers that are to be applied respectively, to different operating conditions of a dynamic nonlinear system (Ahmad and Mohamed, 2009). For the smooth interpolation of linear gains applied across the input space the sugeno fuzzy system is well suited. Three parameters are there in (8). To train these parameters the numbers of input-output training pairs are larger than available parameter. In the computer simulation carried out the training set for each training procedure contains 120 input-output pairs.

Preparation set: The number of available parameters for training the NF controller is:

Input Y(f)_3 MFs *3 pmtrs/MF +inputX(f)_4MFs *3pmtrs /MF+outputDr_12MFs *3pmtrs/MF

$=9$ pmtrs +12 pmtrs +36 pmtrs $=57$ pmtrs

where, $Y(f)$ and $X(f)$ are the inputs with 3 and 4 membership functions and the output is Dr with 12 membership functions. To provide good training the numbers of input-output pairs are larger than the number of available parameters. In the computer simulation carried out, the training set for each training procedure contains 180 input-output pairs. For mapping the intelligent fuzzy controller $(30 \times 30)$ input-output samples are used, the input-output mapping surface of the controller is shown in Fig. 2. The input-output mapping of RBF1 controller is shown in Fig. 3.

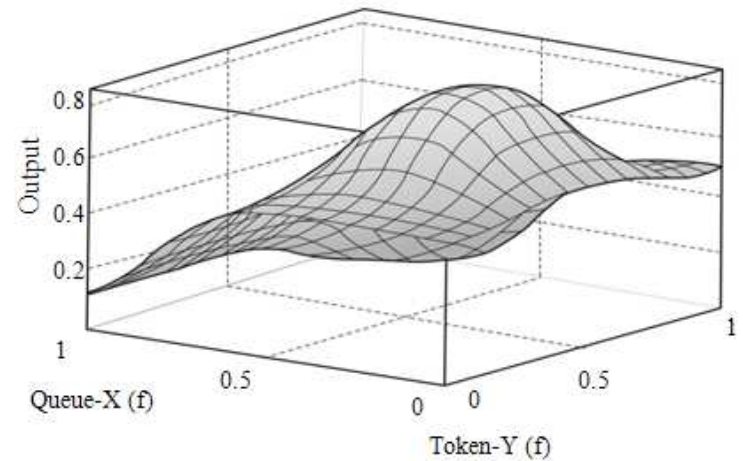

Fig. 2: Nero testing set

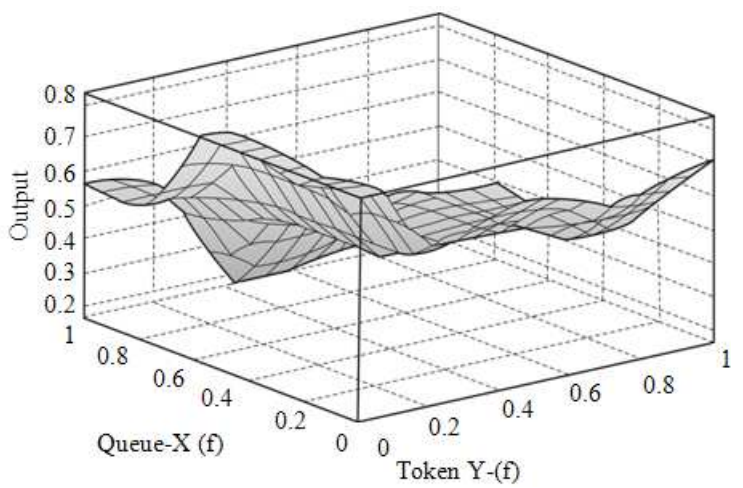

Fig. 3:Input-Output mapping of intelligent fuzzy logic controller

\section{MATERIALS AND METHODS}

The MATLAB function ANFIS (Adaptive NeuroFuzzy Inference System) is used to train the NF controller. The proposed NF controller scheme had been tested on two real- time trailer video Iron baby and Harry potter and the deathly hallows clips. These clips have been resized by deleting all the pixels on the odd columns and rows. Table 2 shows the basic features of the two resized testing clips.

For consistency, the first $300 \mathrm{GOP}_{\mathrm{s}}(300 \times 12=$ 3600 frames) of each clip are presented in the results of Table 4-6. For the wave form analysis of the video clips $200 \mathrm{GOP}_{\mathrm{s}}(200 \mathrm{X} 12=2400$ frames$)$ are presented in Fig. 2.

The video stream data sources need more storage than the sources that flow evenly. However, undesirable delay is caused due to a token bucket with large buffer storage. Considering this; a bucket size of 10 Kbytes is used in the MATLAB SIMULINK computer simulation. The maximum token contract rate Contracr $r_{r}$ is set to $550 \mathrm{~kb} \mathrm{sec}^{-1}$, this produces a maximum acceptable delay of 112 milliseconds. 
Table 1: RBF controller rules

If (Token-Y(f) is empty) and (Queue- $\mathrm{X}(\mathrm{f})$ is empty) then (Output- $\mathrm{d}_{\mathrm{i}}$ is very-large)

If (Token-Y(f) is empty) and (Queue-X(f) is medium) then (Output- $d_{i}$ is very-large)

If (Token-Y(f) is empty) and (Queue-X(f) is full) then (Output- $d_{i}$ is very-large)

If (Token-Y(f) is empty) and (Queue-X(f) is very-full) then (Output- $d_{i}$ is large)

If (Token- $Y(f)$ is medium) and (Queue- $X(f)$ is empty) then (Output- $d_{i}$ is very-large)

If (Token-Y(f) is medium) and (Queue-X(f) is medium) then (Output- $d_{i}$ is intermediate)

If (Token-Y(f) is medium) and (Queue- $X(f)$ is full) then (Output- $d_{i}$ is small)

If (Token-Y(f) is medium) and (Queue- $X(f)$ is very-full) then (Output- $d_{i}$ is very-small)

If (Token-Y(f) is full) and (Queue- $X(f)$ is empty) then (Output- $d_{i}$ is very-large)

10.If (Token- $Y(f)$ is full) and (Queue- $X(f)$ is medium) then (Output- $d_{i}$ is small)

11.If (Token-Y(f) is full) and (Queue-X(f) is full) then (Output- $d_{i}$ is very-small)

12.If (Token-Y(f) is full) and (Queue-X(f) is very-full) then (Output- $\mathrm{d}_{\mathrm{i}}$ is very-small)

Table 2: Test video clips

\begin{tabular}{llll}
\hline Name & Width (pixel) & Height (pixel) & Length (GOP) \\
\hline $\begin{array}{l}\text { Iron baby } \\
\begin{array}{l}\text { Harry potter and } \\
\text { the deathly hallows }\end{array}\end{array}$ & 240 & 124 & 387 \\
\hline
\end{tabular}

Table 3: Wireless channel noise condition Simulated interference $\left[\mathrm{N}\left(e, v^{2}\right)\right]\left(\mathrm{kb} \mathrm{s}^{-1}\right)$

\begin{tabular}{|c|c|c|c|c|}
\hline $\begin{array}{l}\text { Gaussian } \\
\text { distribution }\end{array}$ & $\begin{array}{l}\text { None } \\
{[\mathrm{N}(0,0)]}\end{array}$ & $\begin{array}{l}\text { Low-level } \\
\text { noise } \\
{[\mathrm{N}(0,267)]}\end{array}$ & $\begin{array}{l}\text { High-level } \\
\text { noise } \\
{[\mathrm{N}(0,1112)]}\end{array}$ & $\begin{array}{l}\text { Combined } \\
\text { noise } \\
{[\mathrm{N}(0,267)]+} \\
{[\mathrm{N}(0,1112)]}\end{array}$ \\
\hline $\begin{array}{l}\text { Proposed } \\
\text { system }\end{array}$ & $\begin{array}{l}\text { Experiment } \\
1\end{array}$ & $\begin{array}{l}\text { Experiment } \\
2\end{array}$ & $\begin{array}{l}\text { Experiment } \\
3\end{array}$ & $\begin{array}{l}\text { Experiment } \\
4\end{array}$ \\
\hline
\end{tabular}

This delay duration is less than 3 video frames. In this experiment four different channel noise conditions have been considered. The simulated interference noise of each experiment is shown in Table 3. To examine the maximum bandwidth of wireless when there is no noise present a Non-noise situation is simulated.

In the simulation, $\mathrm{N}\left(\mathrm{m}, \mathrm{v}^{2}\right)$ is used as a Gaussian distribution, where $\mathrm{m}$ is the mean value and $\mathrm{v}$ is the standard deviation. The mean value of the Gaussian interference noise is always zero. The low-level noise has a standard deviation of $\sqrt{267}$ such that $99.7 \%$ of the actual data rate is within the range of [650-700] $\mathrm{kb}$ $\mathrm{sec}^{-1}$. The difference between minimum and maximum token rate is 50 and the maximum acceptable frame delay in the token bucket is 3. In the high-level noise simulation $99.7 \%$ of the data rate is within the range [600-700] $\mathrm{kb} \mathrm{sec}^{-1}$. For high-level noise a standard deviation of $\sqrt{1112}$ is used.

The combination of low-level rapid changing and high-level slow-changing noise is the combined noise. The low-level rapid-changing noise represents the lowlevel interference in the wireless channel. The situation of interfering device like microwave switches on and off is represented in high-level slow-changing noise; the data rate is within the range [500-700] $\mathrm{kb} \mathrm{sec}^{-1}$.

\section{RESULTS}

The results of open loop VBR encoding system are compared with the proposed intelligent NF scheme and are given in Table 4. When the NF scheme is applied the percentage of dropped data decreases by more than $52 \%$ and the variance of output rate of the wireless channel decreases by $82 \%$. The numerical results of testing clips using intelligent fuzzy logic controller is shown in Table 5. The MPEG encoder output variance rate for the open loop VBR system is higher than the NF scheme and RBF1 scheme. In noisy situation like combined noise, the intelligent fuzzy controller adapts itself to the actual channel condition, resulting in an increase in the variance of output rate from the trafficshaper in Table 5, 'Iron baby' clip, $35.65 \mathrm{~kb} \mathrm{sec}^{-1}$.

This is well demonstrated in the graph of Fig. 4d, in the combined noise, Due to the smoothness of the input-output mapping surface of the Sugeno-type NF controller the departure rate $\mathrm{D}_{\mathrm{r}}$ directly follows actual.

\section{DISCUSSION}

From Table 6, the use of intelligent fuzzy logic controller reduces the number of dropped data at the token bucket. With the presence of Gaussian noise 67\% improvement is achieved. For controlling the size and bit rate of the compressed video stream quantization parameters are used in MPEG-4. The image quality is determined by the amount of compression specified by the quantization parameter of the encoder (Rami, 2009), Most of the applied video compression techniques are lossy; they are based on removing redundancy in the spatial, temporal and frequency domains (Abdellaoui, 2009). SIMULINK computer simulation results for Iron baby and Harry potter and the deathly hallows clips are represented in Fig. 4. Each figure has four sets of results for (a) no noise, (b) low noise, (c) high noise and (d) combined noise. Each set has three graphs, the arrival bit rate to traffic-shaper Ar, the departure bit rate from the traffic-shaper Dr and the actual transmission rate actual ${ }_{r}$. 
J. Computer Sci., 7 (7): 1119-1127, 2011
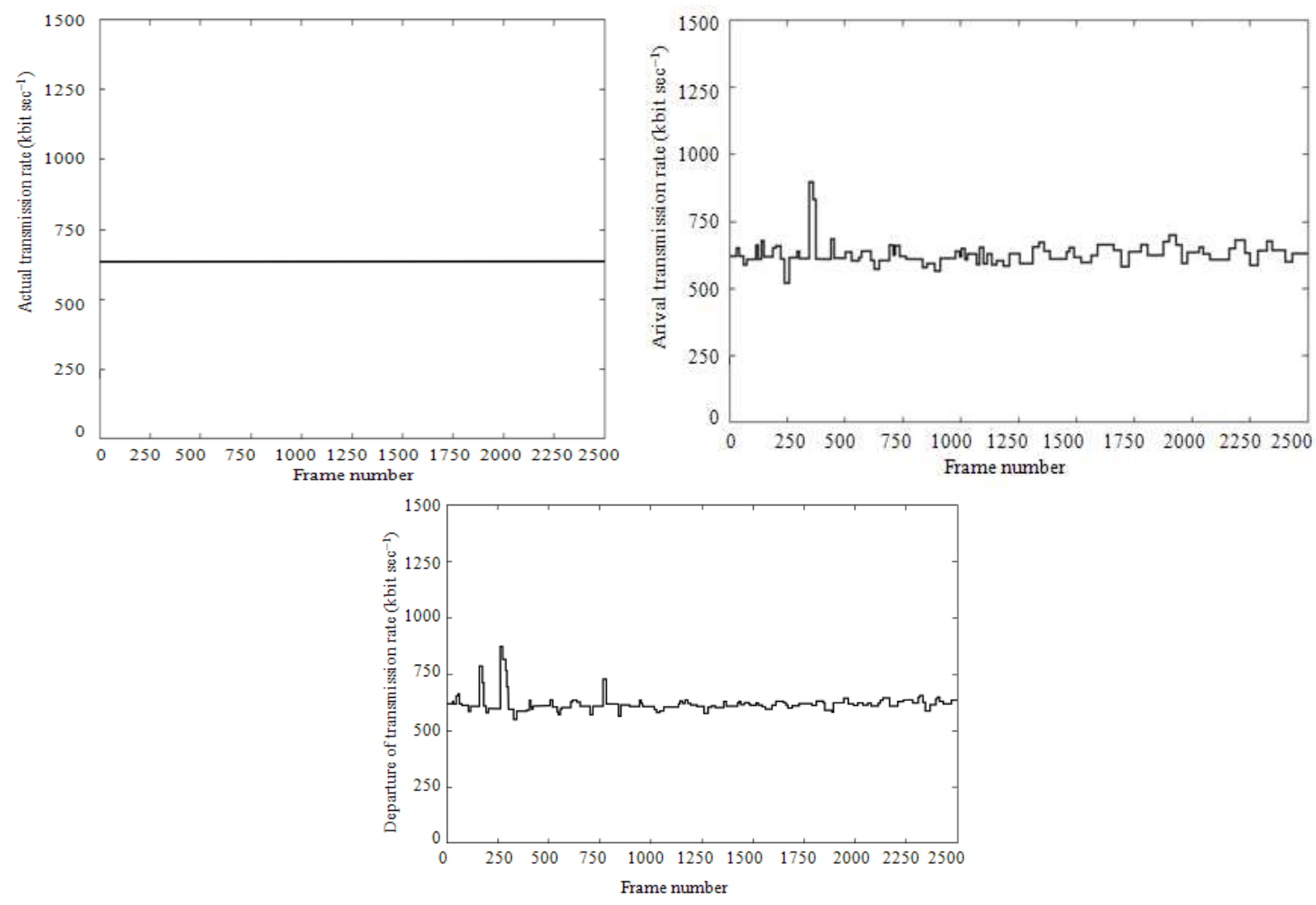

(a)

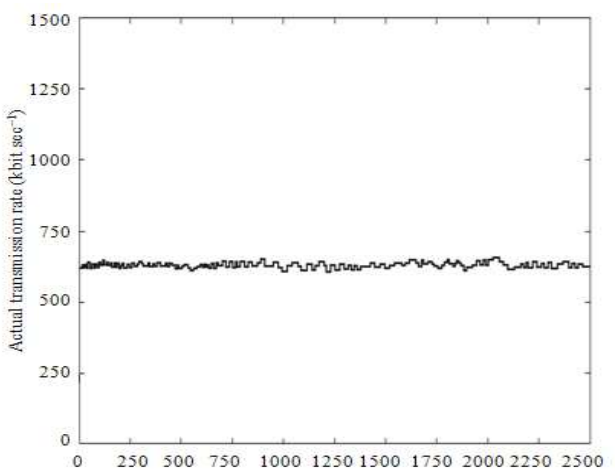

Frame number
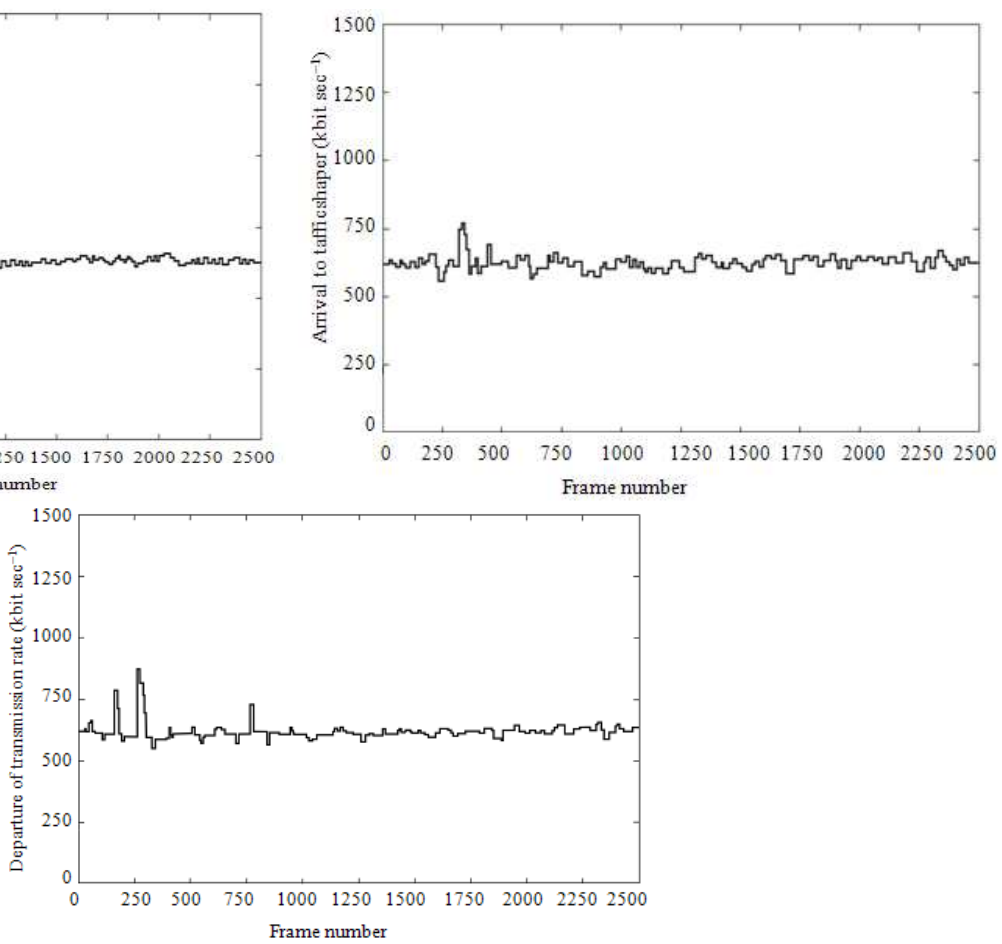

(b)

1124 
J. Computer Sci., 7 (7): 1119-1127, 2011
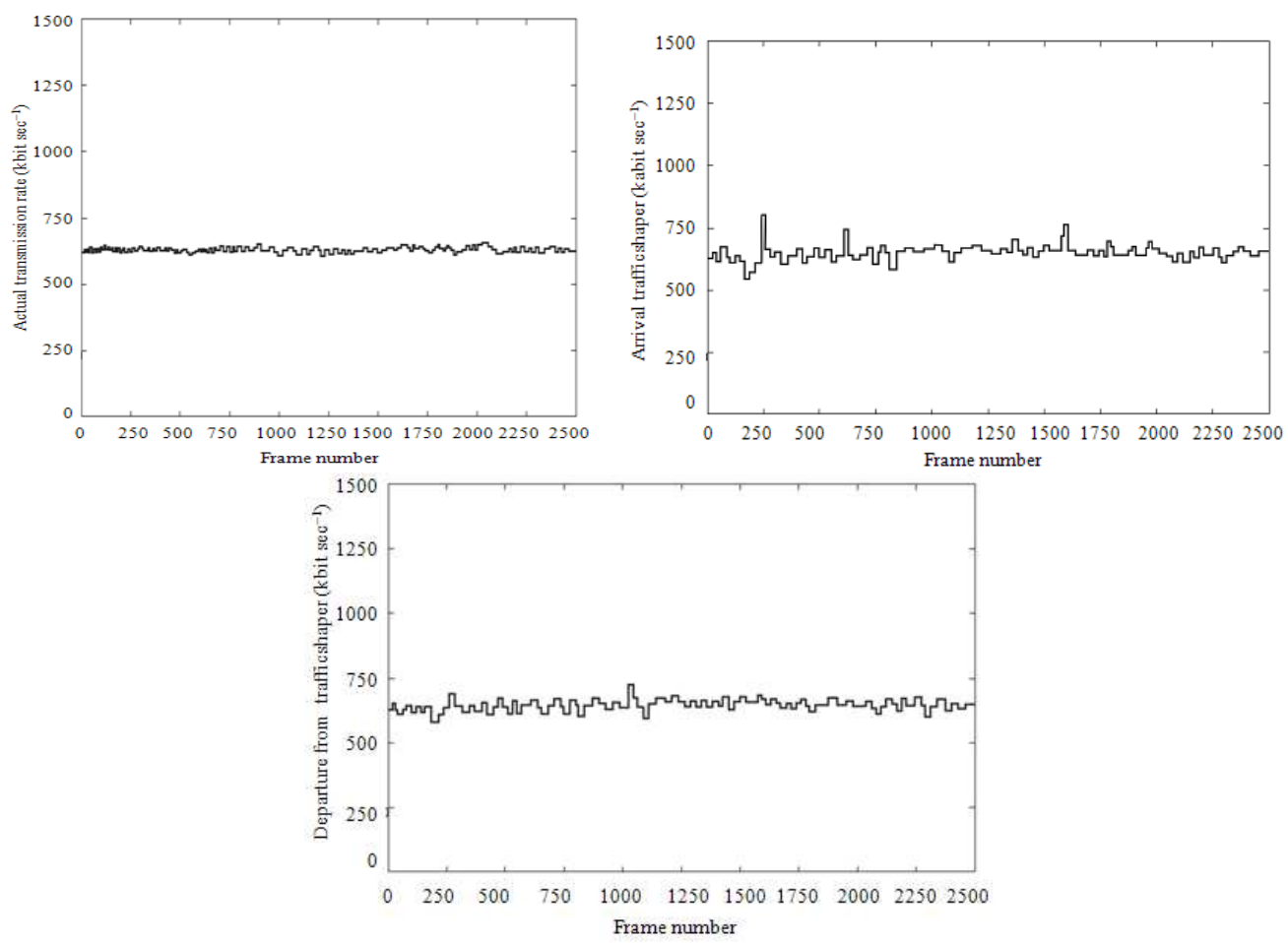

(c)
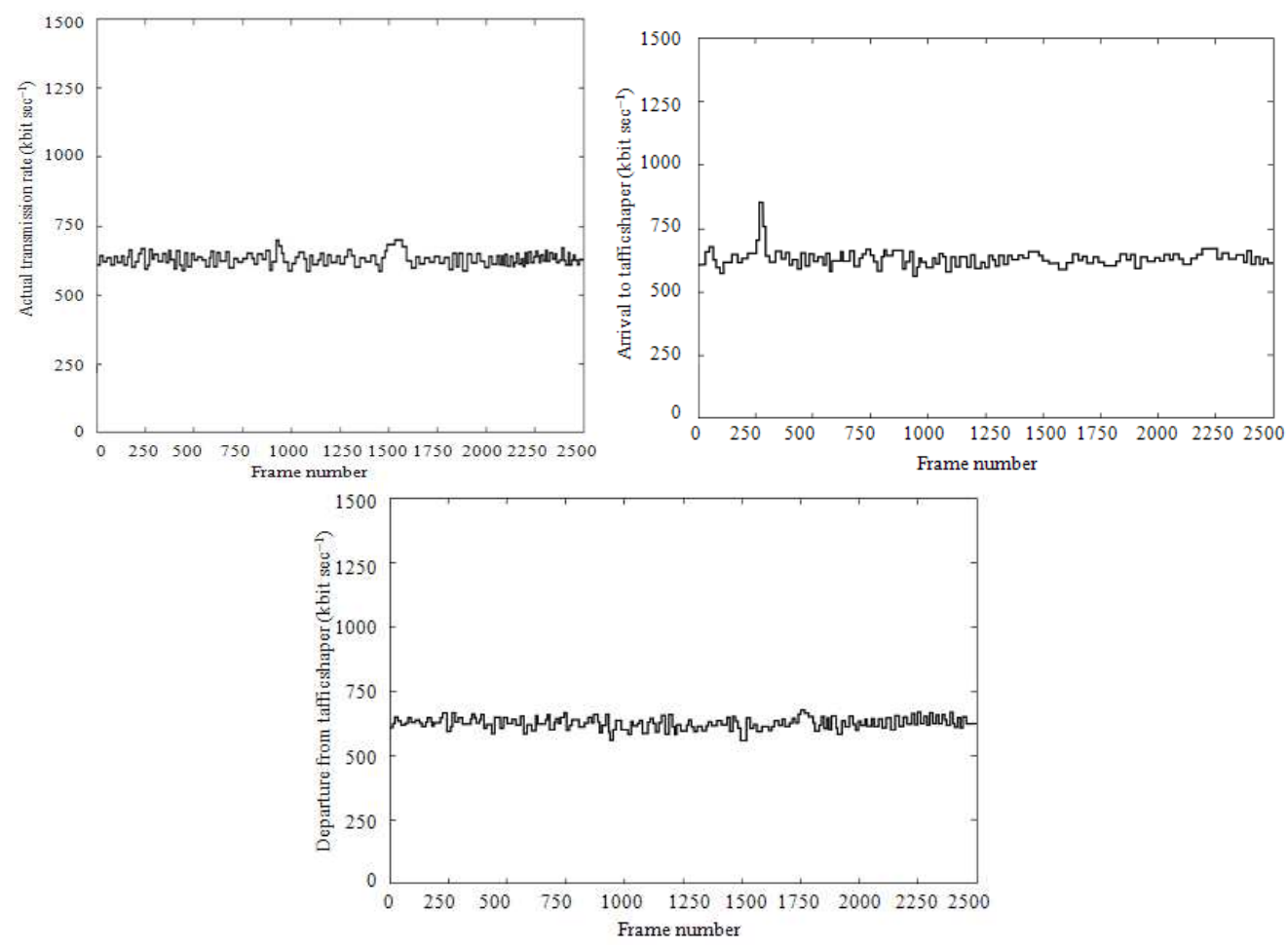

(d)

Fig. 4: Intelligent fuzzy controller with different levels of noise in wireless channel (a) noise of $\mathrm{N}(0,0)$; (b) noise of $\mathrm{N}(0,267)$; (c) noise of $\mathrm{N}(0,1112)$; (d) combined noise of $\mathrm{N}(0,267)+\mathrm{N}(0,1112)$ 
Table 4: Simulation result of test clips

\begin{tabular}{|c|c|c|c|c|c|c|c|c|}
\hline \multirow{2}{*}{$\begin{array}{l}\text { Clip name } \\
\text { Experiment }\end{array}$} & \multicolumn{4}{|c|}{ Iron baby } & \multicolumn{4}{|c|}{ Harry potter and the deathly hallows } \\
\hline & 1 & 2 & 3 & 4 & 1 & 2 & 3 & 4 \\
\hline $\begin{array}{l}\text { Percentage of decrease in variance } \\
\text { of output rate to network by } \\
\text { proposed system }\end{array}$ & 85.3 & 86.4 & 87.5 & 83.2 & 79.2 & 80.6 & 80.1 & 79.6 \\
\hline Percentage of Dropped data & 11.8 & 12.7 & 13.6 & 15.9 & 98.4 & 96.2 & 94.2 & 77.4 \\
\hline $\begin{array}{l}\text { Percentage of Decrease in number } \\
\text { of Dropped data by proposed system }\end{array}$ & 91.4 & 85.1 & 76.5 & 49.2 & 97.7 & 95.3 & 93.2 & 77.7 \\
\hline
\end{tabular}

Table 5: Simulation results using intelligent fuzzy logic controller for iron baby clip

\begin{tabular}{|c|c|c|c|c|}
\hline \multirow[b]{2}{*}{ Experiment } & \multicolumn{4}{|c|}{ Intelligent fuzzy logic controller } \\
\hline & 1 & 2 & 3 & 4 \\
\hline Variance of output rate from MPEG encoder $\left(\mathrm{kb} \mathrm{sec}^{-1}\right)$ & 78.45 & 68.71 & 51.68 & 45.26 \\
\hline Variance of output rate from traffic shaper $\left(\mathrm{kb} \mathrm{sec}^{-1}\right)$ & 31.45 & 28.84 & 25.27 & 35.92 \\
\hline Percentage of dropped data at token bucket & 0.124 & 0.357 & 0.687 & 1.431 \\
\hline Percentage of dropped data at traffic shaper & 0.684 & 1.348 & 2.231 & 6.425 \\
\hline
\end{tabular}

Table 6: Performance of intelligent fuzzy logic controller

\begin{tabular}{llcl}
\hline & Experiment & Iron baby & $\begin{array}{l}\text { Harry potter and the } \\
\text { deathly hallows }\end{array}$ \\
\hline Percentage of & 1 & 6.64 & 16.21 \\
decrease in & 2 & 65.81 & 97.74 \\
number of & 3 & 73.27 & 82.92 \\
dropped data at & 4 & 81.53 & 84.49 \\
token bucket & & & \\
$\begin{array}{l}\text { Percentage of } \\
\text { decrease in }\end{array}$ & 1 & 11.68 & 53.41 \\
total number & 3 & 17.72 & 52.84 \\
of dropped data & 4 & 20.48 & 54.65 \\
& & 7.94 & 43.58 \\
\hline
\end{tabular}

The burstiness of the departure bit rate Dr from the traffic shaper is reduced as compared with the arrival bit rate $A_{r}$ to the traffic shaper, as a result smooth data transmission in the wireless channel. The real-time transmission is uncertain and unpredictable, the NF scheme always adapts to changes encountered by the wireless channel and its environment and the departure rate is increased or decreased to optimize available bandwidth.

\section{CONCLUSION}

The computer simulation results show that, the use of the intelligent fuzzy logic controller reduces the variance of output bit rate to the wireless network. It also reduces number of dropped data and produces better and stable video image quality. The NF controller produces an improved image quality as the adaptive NF scheme adjusts and changes the parameters in the RBF1 controller. Predictions of the network behavior rules in the RBF1 controller are written in advance Table 1 . The intelligent fuzzy logic control scheme also improves and maintains the quality of service with noise interferences over the wireless channel. In real-time video transmission over wireless network, due to external interference of various signals the data are lost. The intelligent fuzzy logic controller readjusts and updates the membership functions to generate its own membership functions from the existing rules during the MPEG-4 VBR video transmission. According to the new occurrence from the wireless channel and its environment, the membership functions in the rules are updated constantly during the transmission period. As a result more MPEG-4 VBR video data can be transmitted. The intelligent fuzzy logic controller can be easily implemented in real-time applications of wireless network; it does not require much power.

\section{REFERENCES}

Abdellaoui, M., 2009. Generic algorithm with varying block sizes to improve the capacity of a wireless communications networks., J. Comput. Sci., 5: 323-329. DOI: 10.3844/JCS.2009.323.329

Ahmad, M.A. and Z. Mohamed, 2009. Hybrid fuzzy logic control with input shaping for input tracking and sway suppression of a gantry crane system. Am. J. Eng. Applied Sci., 2: 241-251. DOI: 10.3844/AJEAS.2009.241.251

Breven, S.P. and N.S.M. El-Tayeb, 2009. A new approach of adaptive network-based fuzzy inference system modeling in laser processing-a Graphical User Interface (GUI) based. J. Comput. Sci., 5: 704-710. DOI: 10.3844/JCSSP.2009.704.710

Chabaa S. and A. Zeroual, 2009. Predicting packet transmission data over IP networks using adaptive neuro-fuzzy inference systems. J. Comput. Sci., 5: 123-130. DOI: 10.3844/JCSSP.2009.123.130 
Chao, C.-H., M.-Y. Hsiao, S.-H. Tsai and T.-H.S. Li, 2010. Design of an interval type-2 fuzzy immune controller. Inform. Tech. J., 9: 1115-1123. DOI: 10.3923/ITJ.2010.1115.1123

Elaal, A.K.A., H.A. Hefny and A.H.A. Elwahab, 2010. Constructing fuzzy time series model based on fuzzy clustering for a forecasting. J. Comput. Sci., $\quad 6: \quad 735-739 . \quad$ DOI: 10.3844/JCSSP.2010.735.739

Emmanuel, A.J., M. Fleury, C. Wagner, H. Hagras and M. Ghanbari, 2009. Interval type-2 fuzzy logic congestion control for video streaming across IP networks. IEEE Transact. Fuzzy Syst., 17: 11231142. DOI: 10.1109 /TFUZZ.2009.2023325

Kazemian, H.B., 2009. An intelligent video Streaming Technique in Zigbee Wireless. Proceedings of the International Conference on IEEE Fuzzy Systems, Aug. 20-24, IEEE Xplore Press, Jeju Island, pp: 121-126. DOI: 10.1109/FUZZY.2009.5277082

Lokman, H.H., M. Moghavvemi and H.A.F. Mohamed, 2010. Takagi-Sugeno fuzzy gains scheduled pi controller for enhancement of power system stability. Am. J. Applied Sci., 7: 145-152. DOI: 10.3844/AJASSP.2010.145.152
Mamdouhi, H. S. Khatun and J. Zarrin, 2009. Bluetooth wireless monitoring, managing and control for inter vehicle in vehicular ad-hoc networks. J. Comput. Sci., $\quad$ 5: $922-929 . \quad$ DOI: 10.3844/JCSSP.2009.922.929

Priakanth, P. and P. Thangaraj, 2009. A channel adaptive energy efficient and fair scheduling media access control protocol for mobile adhoc networks. J. Comput. Sci., 5: 57-63. DOI: 10.3844/JCSSP.2009.57.63

Rami, J.M., 2009. Self-adjustment time quantum in round robin algorithm depending on burst time of the now running processes. Am. J. Applied Sci., 6: 1831-1837. DOI:10.3844/AJASSP.2009.1831.1837

Samimi, A., H.Z. Aashtiani and A. Mohammadian, 2009. A short-term management strategy for improving transit network efficiency. Am. J. Applied Sci., 6: 241-246. DOI: 10.1002/INT.20242

Sasilatha, T and J. Raja, 2009. Comparative studies on the performance of low power transmitters for wireless sensor nodes. J. Comput. Sci., 5: 140-145. DOI: 10.3844/JCSSP.2009.140.145 